\title{
An IoT-Belief Rule Base Smart System to Assess Autism
}

\author{
Md. Eftekhar Alam*, M. Shamim Kaiser ${ }^{\dagger}$, Mohammad Shahadat Hossain ${ }^{\ddagger}$, and Karl Andersson ${ }^{\S}$ \\ *Electrical \& Electronic Engineering, International Islamic University Chittagong, Bangladesh, Email:eftekhar@eee.iiuc.ac.bd \\ †Institute of Information Technology, Jahangirnagar University, Dhaka, Bangladesh, Email: mskaiser@ juniv.edu

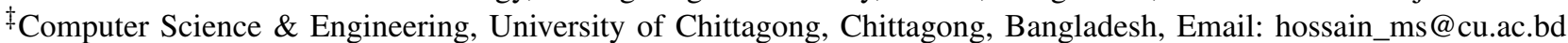 \\ §Pervasive and Mobile Computing Laboratory, Luleå University of Technology, Skellefteå, Sweden, Email:karl.andersson@ltu.se
}

\begin{abstract}
An Internet-of-Things (IoT)- Belief Rule Base (BRB) based hybrid system is introduced to assess Autism spectrum disorder (ASD). This smart system can automatically collect sign and symptom data of various autistic children in realtime and classify the autistic children. The BRB subsystem incorporates knowledge representation parameters such as rule weight, attribute weight and degree of belief. The IoT-BRB system classifies the children having autism based on the sign and symptom collected by the pervasive sensing nodes. The classification results obtained from the proposed IoT-BRB smart system is compared with fuzzy and expert based system. The proposed system outperformed the state-of-the-art fuzzy system and expert system.
\end{abstract}

\section{INTRODUCTION}

Autism spectrum disorder (ASD) is a neurologi$\mathrm{cal} /$ developmental disorder which infects behavior and communication of a person. The symptoms of this disorder come into sight in the first two years of a child. The signs mainly appear in the spoken language and the social interactions with the others. However the symptom may include a learning disability, hyperactivity, epilepsy, obsessive compulsive disorder, bipolar disorder, anxiety disorder, bipolar disorder, and sensory difficulties [1], [2], [3], [4].

Various statistical portal illustrates the ASD related publication, their appearance in the school are raising worldwide. The US department of health and human services has found that there is at least 1 in 68 persons challenged by an ASD. Unfortunately, no data has been found for how many people have ASD in Bangladesh [1], [5], [2].

A significant number of research works have been reported to identify Autism. Alharbi et al. [1] proposed the Belief Rule-Based Expert System (BRBES) where rule base with uncertainty is used for representing the data. It deals with different kinds of vulnerability related to the autism factors and considered as learning representation parameters, such as rule weight which assumes a vital part in expanding the quality of the framework result. Authors showed the BRBES performed better than expert option and fuzzy based framework. Moreover, the framework permits the age of different 'what if' scenarios which can be used to build up a suitable treatment plan for the mentally unbalanced children. Thus, this framework can be used to assess the condition of the autistics children. However, the system has collected data manually.
Najwani et al [2] proposed Gaussian mixture model (GMM) and Multilayer perceptron (MLP) based classifier to identify autistic children based on Electroencephalography (EEG) signal. Experimental result showed the potential of verifying between autistic and normal children with accuracy of $92 \%$. This method can be used to identify autistic children which can help early detection for the purpose of early intervention. Moreover, the spectrum of the signals also present big differences between the two groups. The system automatic collection of data by a sensor network.

Maite et al [6] proposed a framework which considered qualitative and quantitative measures and mainly differentiated and characterized the levels of severity of the phonological disorder of an autistic child. The outcomes are spoken to as a bar diagram with the percent score of the right elocution of each word. These outcomes can be traded and put away to keep a fleeting register of definite client's movement. However, uncertainty can be caused by the system. The performance can be fastened with the help of Internet of things (IoT).

Arthi and Tamilarasi [7] presented a neuro fuzzy based system for the prediction of autistic disorder. The system inputted the physiological data manually.

Sula et al. [8] implemented a IoT based peer-to-pear (P2P) system for supporting Autism Spectrum Disorder (ASD). The authors found that the proposed system has improved the life of autistic people in various ways.

Based on the above discussion, it can be summarized that IoT based expert system is required to assess autism under uncertainty. In contrast to Artificial Neural Network, Support vector machine and Fuzzy based expert system, a Belief Rule Based (BRB) Expert System is able to handle data with uncertainty.

Thus, a IoT-BRB smart system is proposed in this paper to assess ASD under uncertainty.

The rest of the paper is organized as follows. Section II reviewed the IoT and BRB system. An overview of IoT and Belief Rule Based Inference Methodology using the Evidential Reasoning (RIMER) approach, system architecture, design and implementation of the proposed IoT-BRB Smart system are discussed. Experimental results and discussions are are presented in Section III. The hardware and software component of the proposed prototype system is discussed in 
Section IV. Section V concluded this work and summarize the contribution.

\section{OVERVIEW OF IOT AND BRB SYSTEM}

Figure 1 shows a proposed scenario where IoT nodes (with three sensors: Ear-clip heart rate sensor, EMG (Electromyography) sensor and microphone) collect physiological and behavioural data. Then data will be analyzed which includes bigdata, cleansing, streaming technique and storage of data. Finally the ASD classification will be employed by using data mining, machine learning technique.

A block diagram of IoT-BRB system is shown in Figure 2. Here data analysis block indicates the collected data is being analyzed and stored the information. The data Abstraction block aggregates the processed results and converted into actionable knowledge using the BRB Smart system[9].

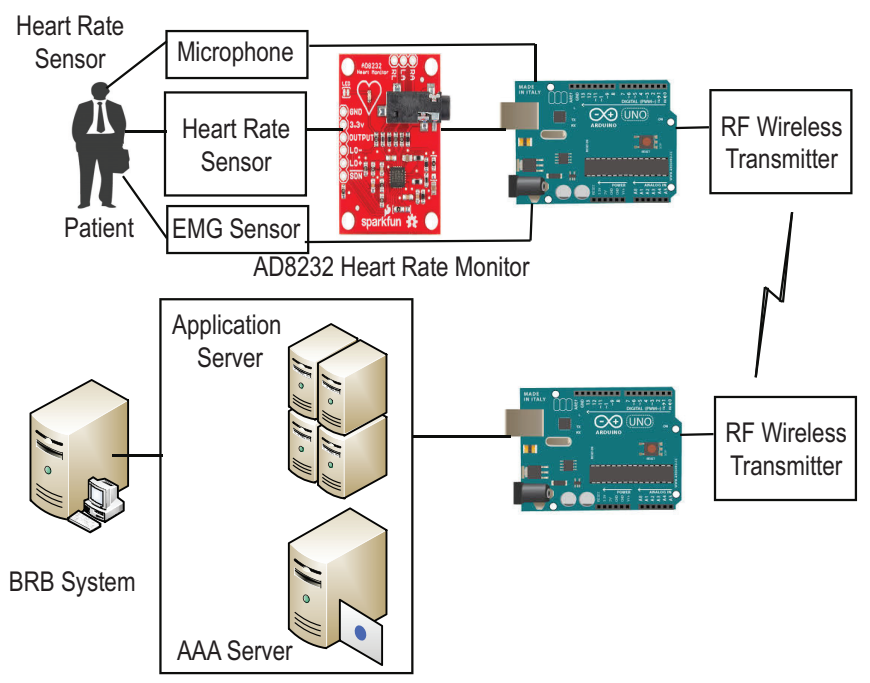

Fig. 1. A proposed scenario

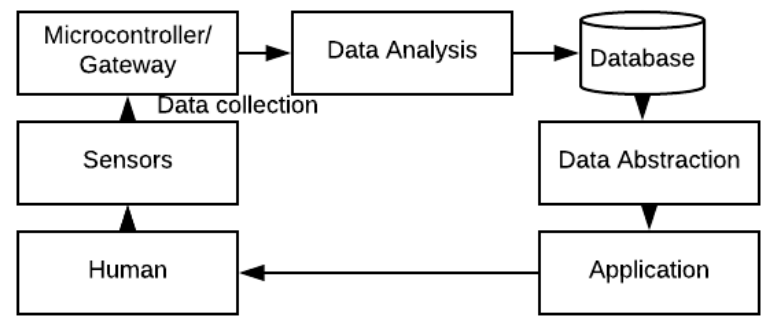

Fig. 2. IoT-BRB system architecture describing functionalities of various layers.

In this section BRB and IoT systems have been discussed.

\section{A. IoT}

A Internet of Things is a network of items where each item is embedded with sensors, software, electronics. These item can be connected to the internet.
Figure 1 illustrates the architecture of the WSN, used to collect and store data from the body of the autistic children. Ear-clip sensor, EMG sensor as well as Microphone have been considered in each IoT node .

In each IoT node, three sensors have been used, these are microphone, heart rate and EMG sensors. The microphone can collect Behaviour, Communication, Social interaction data; the EMG sensor can collect motor data and Ear-clip heart rate sensor collects heart rate data from each subject. The sensor data are stored in the SD card of Arduino and after preprocessing, these data are transferred into database server via RF module connected to the Arduino shield. The sensor data stored in the database are then passed into Web-based BRB smart system.

\section{B. $B R B$}

A BRB system is an supplement to the traditional rule based system which represents more complicated causal relationships considering various kinds of information with uncertainties [10], [11], [12]. A belief degree is associated with each possible consequence of a rule in a Belief rule-base expert system. The knowledge base of the expert system is developed using the belief rules while evidential reasoning approach which is the replacement of inference engine in the expert system. The complicated and continuous causal relationships between different factors can be captured by belief rule-base expert system which is not possible in traditional IF-THEN rules. Like the Fuzzy logic, the BRB system has three parts: an antecedent part, belief rule base and a consequent part. The antecedent parameters take referential values whereas each consequent refers to the degree of belief[13], [14], [15].

The strength of BRB over other schemes is designed with belief degrees attached with all possible terms of a rule in order to capture the causes of uncertainty.

In this approach, a rule base is designed with belief degrees embedded in all possible consequents of a rule. Such a rule base is able to remove vagueness, incompleteness from various factors involved during autism assessment. Fig. 3 illustrates the main components of the Belief Rule Base architecture. The proper interaction of these components creates a perfect BRB and ER based system which is called RIMER methodology [1]. Each of the components of the BRB architecture is elaborated below [16]:

- Presentation layer: This layer is concerned with ensuring the interaction between the users and the system. The users of BRB can interact with the system by using this layer and hence, it works as the interface of the BRBES. Fig 4. illustrates the user interface of the BRBES.

- Application layer: The application layer easily moves data between the computer and the system itself. Application layer cooperates with the deduction motor and preparing module of this BRB framework structure. The BRB induction arrangement of this engineering comprises of a few components, for example, input change, administer actuation weight estimation, govern refreshing instrument, trailed by the total of the principles of a BRB. 
- Data processing layer: This layer is concerned with storing the belief rule base as well as the sensor data received from various sensors as discussed before.

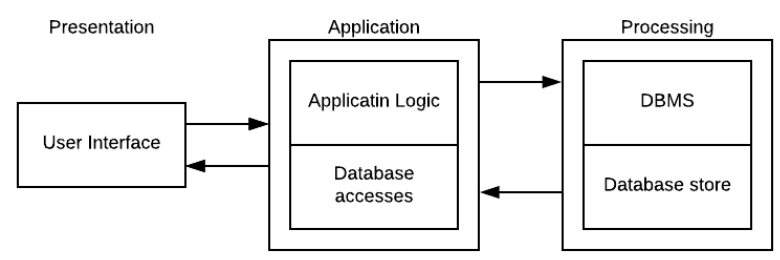

Fig. 3. A BRB Smart Architecture .

The BRBES has been used to measure the level of autism. This system generates results considering various signs and symptoms associated with autism. Signs are directly discovered by physicians and symptoms are obtained from patients experience and feelings. A specialized system or structure is a computer program that imitates human expert which manages clear and compact expertise through wireless sensor network that is capable to explain real-world practical difficulties and work as a value effective adviser. Wireless sensor networks collect real data from the autistic children which help us to measure the level of autism. Main challenges in data collection in wireless sensor networks are sensor memory capacity, energy efficiency, and data storage mechanism. In static sink approach where sink node is static so that energy efficiency is an important problem. Network lifetime is also reduced in such cases. Data storage mechanism is an important challenge in data collection. Good data storage mechanism can also improve the quality of collected packets [15].

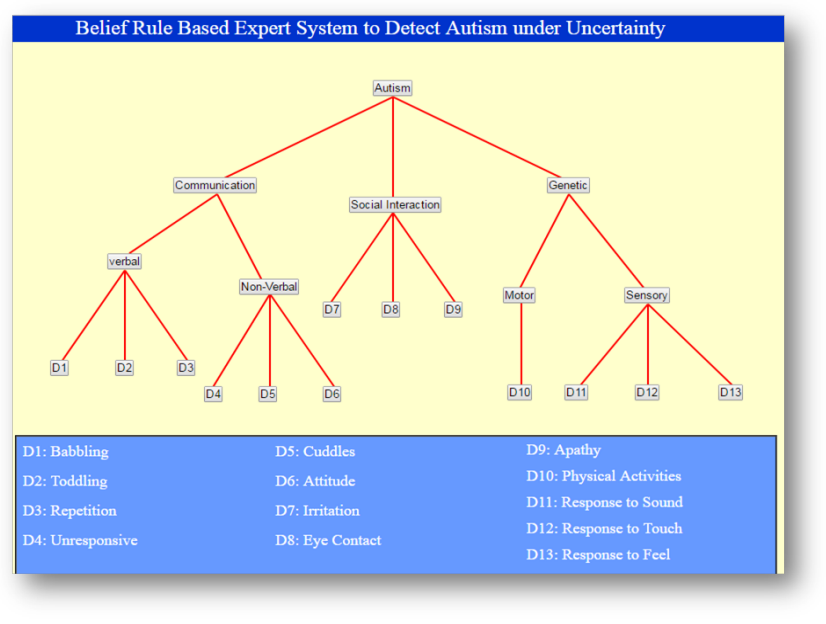

Fig. 4. BRB Interface

The BRB interface is shown in Figure 4 , where degree of belief for the mid level node of the BRB tree calculated in terms of fuzzy value. The overall assessment of the autism of a child has been obtained in terms of crisp value.

\section{A System SetuP}

The following hardware components and software are used for implementing a prototype IoT-BRB Smart system. The hardware components are:

- Arduino(UNO)

- Sensors: Ear-clip Heart Rate Sensor, EMG sensor and Microphone

- RF Wireless Encoder/Decoder

- Arduino Ethernet Shield B

The software are

- Arduino IDE

- java

- LISP (List Processing)

- PROLOG (Program logic)

- PHP

- JQUERY

- Database: MySQL

A photograph of the practical implementation of the proposed prototype system is shown in Figure 5

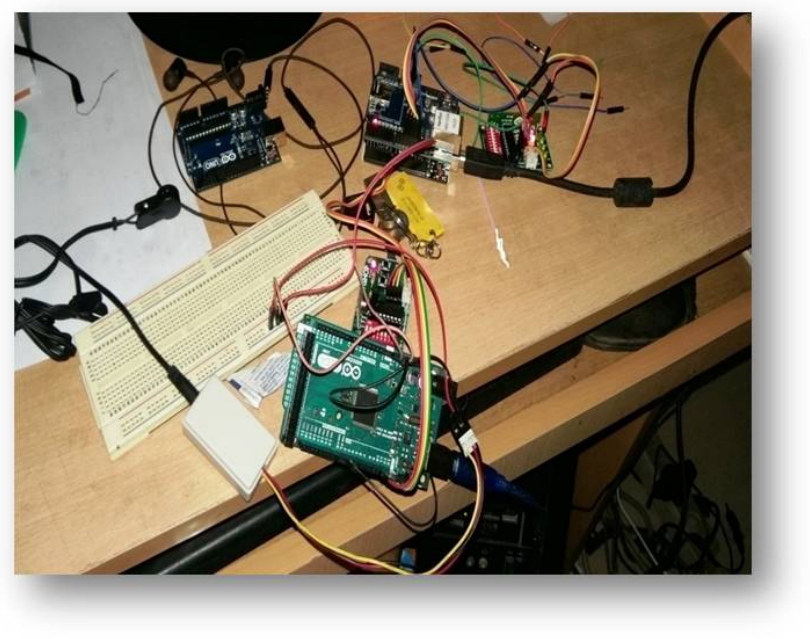

Fig. 5. The practical implementation of the proposed IoT-BRB system.

\section{NumERICAL RESUlts}

The verbal, non-verbal, social interaction, motor and sensory data of different subjects (students at the autistic school located in Chittagong city of Bangladesh) have been collected using IoT sensor nodes. The collected data are then analyzed by BRB system.

\section{A. Data Collection}

The data has been collected from 10 subjects after taking ethical consent. All the subjects are termed by a number and the privacy of the data has been maintained.

In each IoT node, three sensor's have been used, these are microphone and Ear-clip Heart rate sensor, EMG sensors. The microphone can collect Behaviour, Communication, Social interaction data whereas the Ear-cilp Heart rate and EMG 


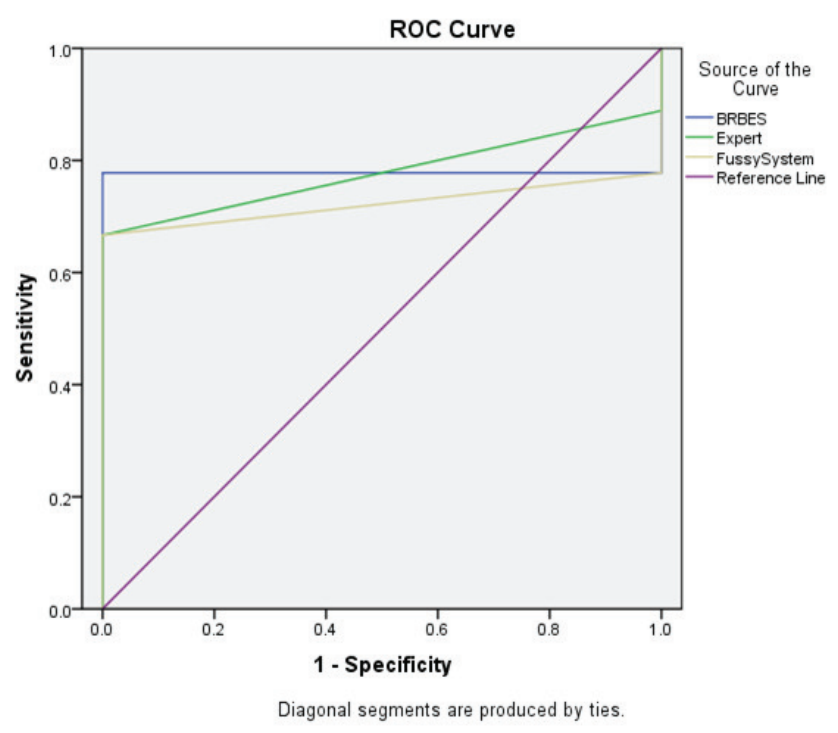

Fig. 7. ROC curve representing BRBES, Experts assumption and Fuzzy output

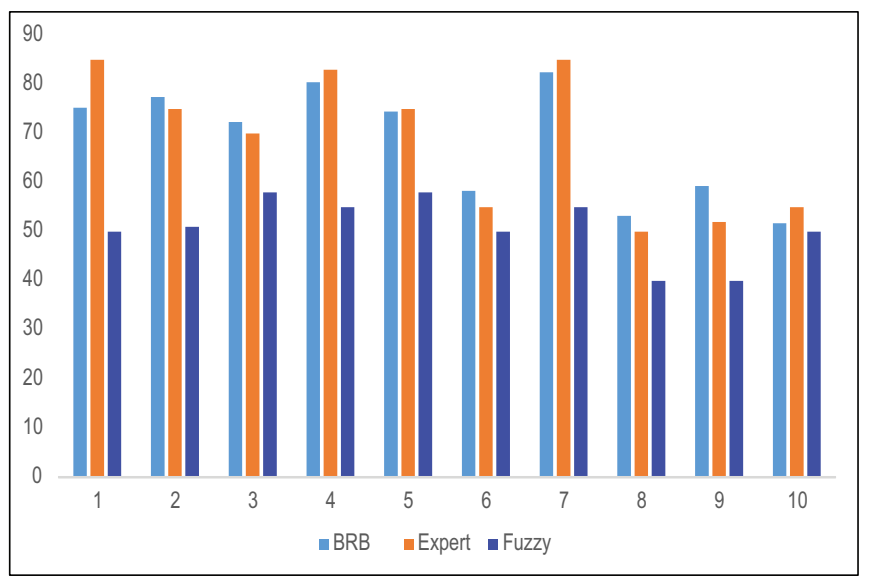

Fig. 6. Autism Assessment both by BRB, Expert and Fuzzy

TABLE I

AUTISM THOUGHT BY BRBES AND EXPERTS

\begin{tabular}{|c|c|c|c|c|c|c|}
\hline Student ID & V & Non-V & SI & M & BRB & Expert \\
\hline 1 & 55 & 48 & 59 & 83 & 75.23 & 75 \\
\hline 2 & 50 & 55 & 49 & 78 & 77.43 & 75 \\
\hline 3 & 78 & 57 & 55 & 52 & 72.33 & 70 \\
\hline 4 & 75 & 34 & 88 & 92 & 80.44 & 78 \\
\hline 5 & 57 & 90 & 43 & 56 & 74.44 & 75 \\
\hline 6 & 89 & 55 & 82 & 72 & 58.34 & 55 \\
\hline 7 & 92 & 53 & 95 & 94 & 82.47 & 85 \\
\hline 8 & 40 & 29 & 55 & 55 & 53.22 & 55 \\
\hline 9 & 49 & 52 & 55 & 82 & 59.31 & 52 \\
\hline 10 & 21 & 48 & 45 & 89 & 51.72 & 53 \\
\hline
\end{tabular}

sensors can collect heart rate and motor data from each subject respectively.

\section{B. Data Analysis and Knowledge Discovery}

These data are then fed into the BRB system to assess the autism spectrum of each subject.

The effectiveness of the assessment are then further analyzed by the Receiver Operating Characteristic (ROC) curve. The performance of the proposed system is calculated using the Area Under Curve (AUC). It has been found that AUC of the proposed system is higher than Fuzzy system.

Figure 7 illustrates ROC curves which are performance of the BRBs, Expert system and Fuzzy system. The ROC curve with blue line indicates the expert opinion while the curve with green line shows the BEB result. The AUC for BRB is higher than that expert opinion.

Table I shows the performance comparison of the BRB and Expert system. It has been found that the reliability of the proposed IoT based BRB smart system is higher than its counterpart.

\section{CONCLUSION}

In this paper, we proposed IoT-BRB based real-time system to assess ASD. The system can not only handle various uncertainty factors exist in a autistic children but also incorporate knowledge representation parameters (such as rule weight, attribute weight and degree of belief). The results obtained from the proposed IoT-BRB baed smart system outperformed stateof-the-art fuzzy/expert based system. It means the proposed system is robust and can be used to develop an appropriate treatment plan for the autistic children. In future, more sensors can be employed to improve the reliability and detection accuracy of ASD by the proposed system .

\section{REFERENCES}

[1] M. S. H. Saad Talal Alharbi and A. A. Monrat, "A belief rule based expert system to assess autism under uncertainty," in Lecture Notes in Engineering and Computer Science : Proceedings of The World Congress on Engineering and Computer Science 2015, Dec 2015, pp. 483-490.

[2] N. Razali and A. W. A. Rahman, "Motor movement for autism spectrum disorder (asd) detection," in Proceeding of the 3rd International Conference on Information and Communication Technology for the Moslem World (ICT4M) 2010, Dec 2010, pp. E90-E95.

[3] M. Randolph-GIps, "Autism: A systems biology disease," in 2011 IEEE First International Conference on Healthcare Informatics, Imaging and Systems Biology, July 2011, pp. 359-366.

[4] R. Ul Islam, M. S. Hossain, and K. Andersson, "A novel anomaly detection algorithm for sensor data under uncertainty," Soft Comput., vol. 22, no. 5, pp. 1623-1639, Mar. 2018. [Online]. Available: https://doi.org/10.1007/s00500-016-2425-2

[5] W. Editor, "Global Autism Movement and Bangladesh," Sep. 2014. [Online]. Available: http://cri.org.bd/2014/09/03/global-autismmovement-and-bangladesh/

[6] M. Frutos, I. Bustos, B. G. Zapirain, and A. M. Zorrilla, "Computer game to learn and enhance speech problems for children with autism," in 2011 16th International Conference on Computer Games (CGAMES), July 2011, pp. 209-216.

[7] K. Arthi and A. Tamilarasi, "Prediction of autistic disorder using neuro fuzzy system by applying ann technique," International Journal of Developmental Neuroscience, vol. 26, no. 7, pp. $699-704$, 2008. [Online]. Available: http://www.sciencedirect.com/science/article/pii/S0736574808001184

[8] M. Randolph-GIps, "Autism: A systems biology disease," in 2011 IEEE First International Conference on Healthcare Informatics, Imaging and Systems Biology, July 2011, pp. 359-366. 
[9] M. S. Hossain, K. Andersson, and S. Naznin, "A belief rule based expert system to diagnose measles under uncertainty," in Proceedings of the 2015 International Conference on Health Informatics and Medical Systems (HIMS'15) :, 2015, pp. 17-23, godkänd; 2015; 20150525 (karand). [Online]. Available: http://www.world-academy-of-science.org

[10] M. S. Hossain, F. Ahmed, Fatema-Tuj-Johora, and K. Andersson, "A belief rule based expert system to assess tuberculosis under uncertainty," Journal of Medical Systems, vol. 41, no. 3, p. 43, Jan 2017. [Online]. Available: https://doi.org/10.1007/s10916-017-0685-8

[11] M. S. Hossain, M. S. Khalid, S. Akter, and S. Dey, "A belief rule-based expert system to diagnose influenza," in 2014 9th International Forum on Strategic Technology (IFOST), Oct 2014, pp. 113-116.

[12] M. S. Hossain, S. Rahaman, R. Mustafa, and K. Andersson, "A belief rule-based expert system to assess suspicion of acute coronary syndrome (acs) under uncertainty,' Soft Computing, Jul 2017. [Online] Available: https://doi.org/10.1007/s00500-017-2732-2

[13] K. Andersson and M. S. Hossain, "Smart risk assessment systems using belief-rule-based dss and wsn technologies," in 2014 4th International
Conference on Wireless Communications, Vehicular Technology, Information Theory and Aerospace Electronic Systems (VITAE), May 2014, pp. $1-5$.

[14] R. Ul Islam, K. Andersson, and M. S. Hossain, "A web based belief rule based expert system to predict flood," in Proceedings of the 17th International Conference on Information Integration and Web-based Applications \& Services, ser. iiWAS '15. New York, NY, USA: ACM, 2015, pp. 3:1-3:8. [Online]. Available: http://doi.acm.org/10.1145/2837185.2837212

[15] M. S. Hossain, P.-O. Zander, M. S. Kamal, and L. Chowdhury, "Belief-rule-based expert systems for evaluation of e-government: a case study," Expert Systems, vol. 32, no. 5, pp. 563-577. [Online]. Available: https://onlinelibrary.wiley.com/doi/abs/10.1111/exsy.12110

[16] M. S. Hossain, S. Rahaman, A. Kor, K. Andersson, and C. Pattinson, "A belief rule based expert system for datacenter pue prediction under uncertainty," IEEE Transactions on Sustainable Computing, vol. 2, no. 2, pp. 140-153, April 2017. 